Supporting Information

\title{
Uniform and Reliable Dip-Coated Conjugated Polymers for Organic Transistors as Obtained by Solvent Vapor Annealing
}

Gun Woo Kim ${ }^{\dagger}$, Eun Hye Kwon ${ }^{\dagger}$, Min Kim*,* and Yeong Don Park ${ }^{*}, \uparrow \S$

$\dagger$ Department of Energy and Chemical Engineering and ${ }^{\S}$ Innovation Center for Chemical Engineering, Incheon National University, Incheon 22012, Republic of Korea

* Center for Nano Science and Technology @ Polimi, Istituto Italiano di Tecnologia, Via Giovanni Pascoli 70/3, 20133 Milan, Italy

*E-mail: min.kim@iit.it (M.K.).

*E-mail: ydpark@inu.ac.kr (Y.D.P.). 
(a) Withdrawn from CF

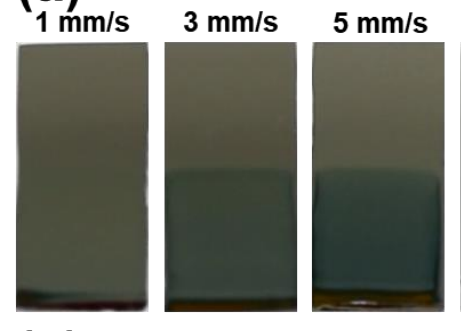

(c) Withdrawn from CF

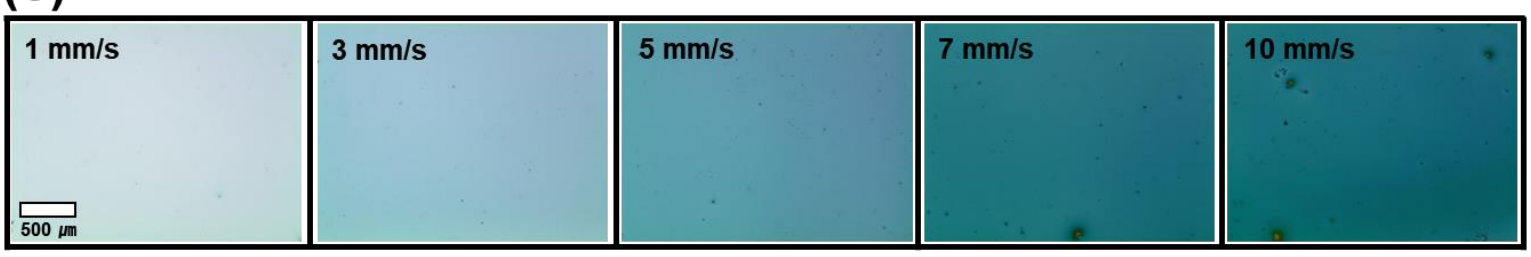

(d) Residual time after withdrawal at $1 \mathrm{~mm} / \mathrm{s}$

\begin{tabular}{|l|l|l|l|l|}
\hline $0 \mathrm{~s}$ & $10 \mathrm{~s}$ & $20 \mathrm{~s}$ & $30 \mathrm{~s}$ & $40 \mathrm{~s}$ \\
\hline $500 \mathrm{um}$ & & & & \\
\hline
\end{tabular}

Figure S1. (a) Photographs of P3HT films dip-coated from chloroform (CF) at the withdrawal speed from $1 \mathrm{~mm} \mathrm{~s}^{-1}$ to $10 \mathrm{~mm} \mathrm{~s}^{-1}$. (b) Photographs of P3HT films dip-coated from CF at the withdrawal speed of $1 \mathrm{~mm} \mathrm{~s}^{-1}$ with various residual time from $0 \mathrm{~s}$ to $40 \mathrm{~s}$. (c) Optical microscopy images of P3HT films dip-coated from chloroform $(\mathrm{CF})$ at the withdrawal speed from $1 \mathrm{~mm} \mathrm{~s}^{-1}$ to $10 \mathrm{~mm} \mathrm{~s}^{-1}$. (d) Optical microscopy images of P3HT films dip-coated from CF at the withdrawal speed of $1 \mathrm{~mm} \mathrm{~s}^{-1}$ with various residual time from $0 \mathrm{~s}$ to $40 \mathrm{~s}$. 
(a)

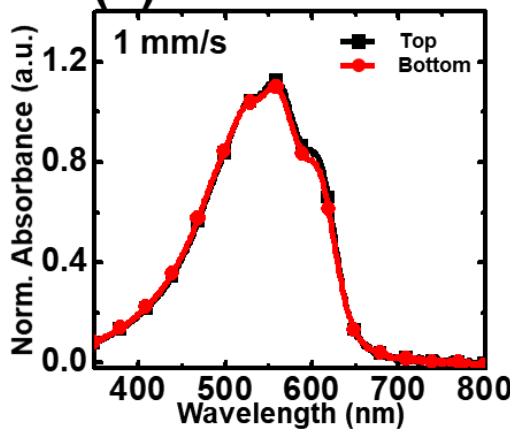

(d)

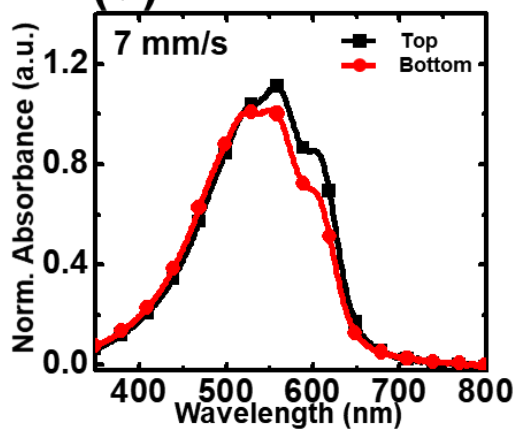

Processed from CB solution

(b)

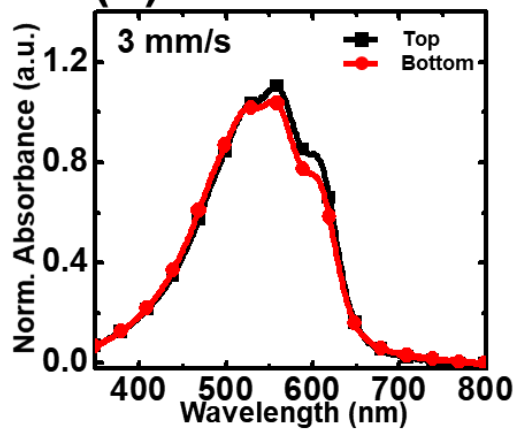

(e)

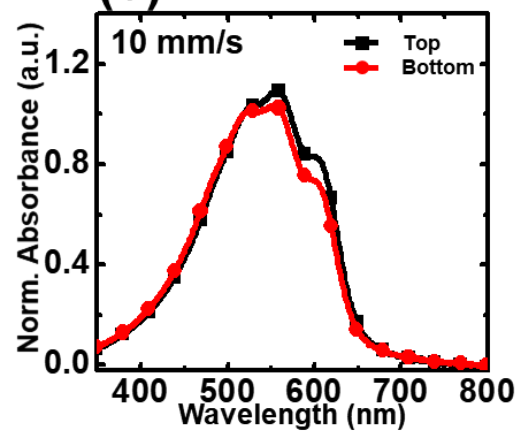

(c)

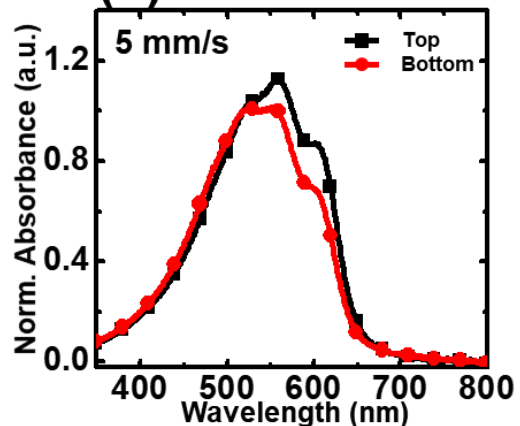

(f)

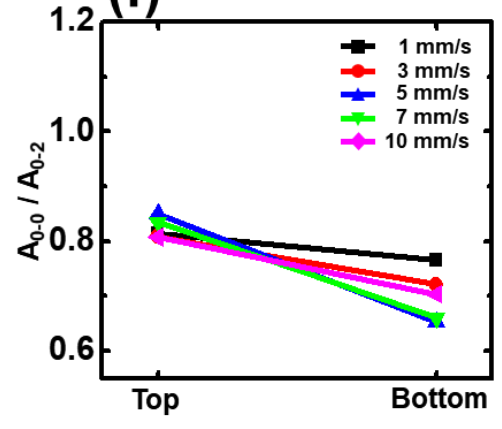

Figure S2. Normalized UV-vis absorption spectra of the P3HT thin films prepared by dip-coating in chlorobenzene (CB) solution with a residual time of $0 \mathrm{~s}$ and withdrawn at (a) 1, (b) 3, (c) 5, (d) 7, and (e) $10 \mathrm{~mm} \mathrm{~s}^{-1}$; at the designated position from the top to the bottom. (f) The ratios of the intensities of the (0-0) to (0-2) peaks as functions of the withdrawal speeds of the dip-coated films. Unlike the dip-coated P3HT films from CF solution, the films withdrawn from CB solution show not only a similar values of the (0-0)/(0-2) peak ratios between the relative vertical positions and but also weak differences in the (0-0)/(0-2) peak ratios between various withdrawal speeds because the vapor pressure of $\mathrm{CB}$ is lower than that of $\mathrm{CF}$. 
(a)

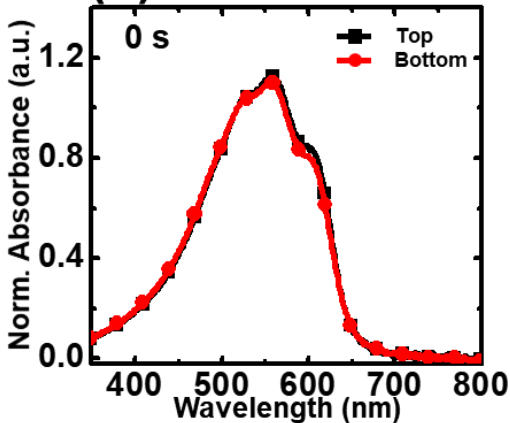

(d)

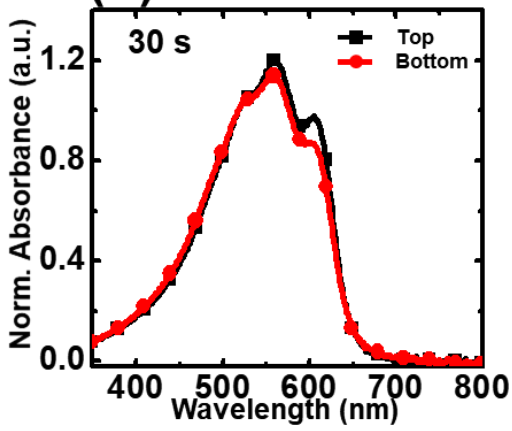

Processed from CB solution

(b)
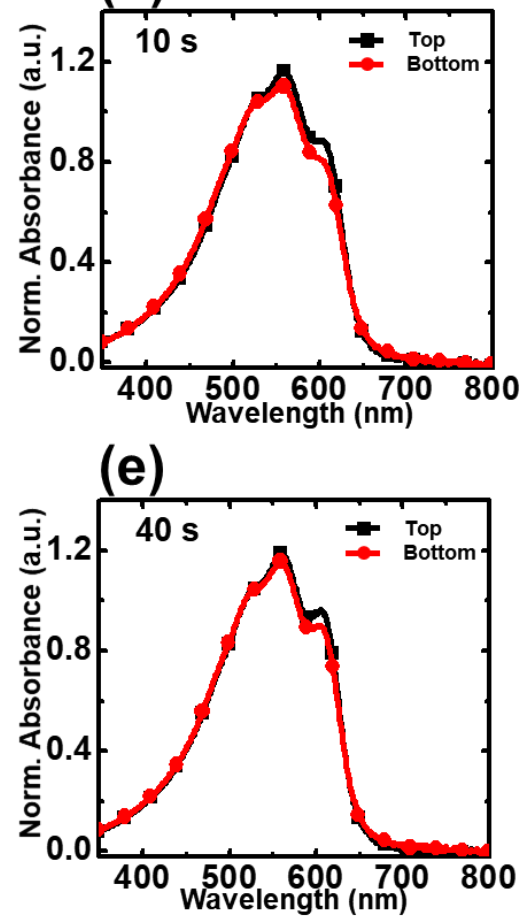

(c)

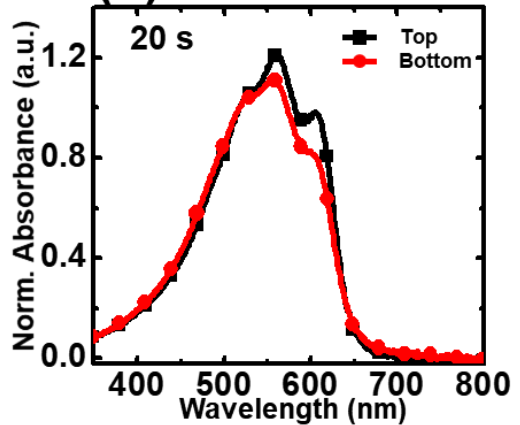

(f)

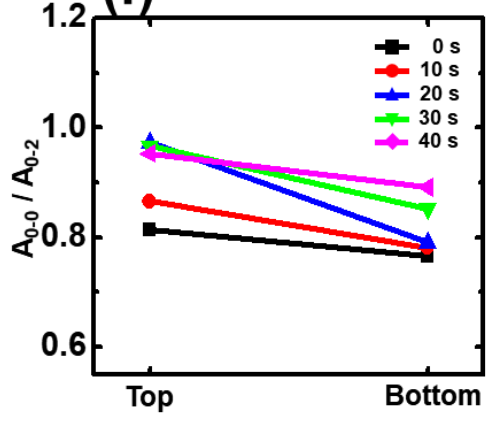

Figure S3. Normalized UV-vis absorption spectra of the P3HT thin films deposited from CB solution at a withdrawal speed of $1 \mathrm{~mm} \mathrm{~s}^{-1}$ with various residual times ranging from (a) 0 , (b) 10 , (c) 20, (d) 30, and (e) $40 \mathrm{~s}$, as recorded at the designated positions from the top to bottom. (f) The ratios of the intensities of the (0-0) to (0-2) peaks as a function of the residual time in the dipcoating chamber. 

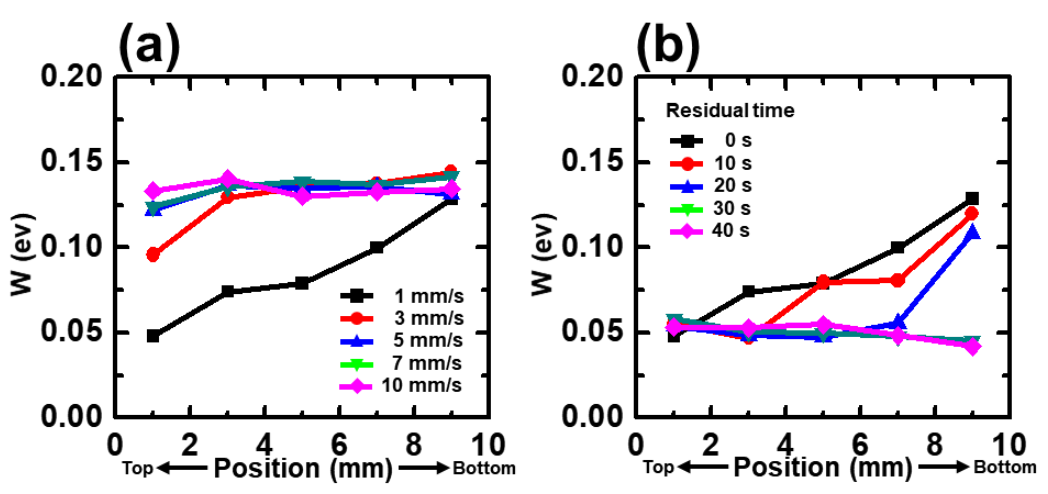

Figure S4. Variations in the free exciton bandwidth (W), calculated from the UV-vis absorption spectra of the dip-coated P3HT thin films from CF solution (a) at various withdrawal speed $($ residual time $=0 \mathrm{~s})(\mathrm{b})$ with residual time from 0 to $40 \mathrm{~s}$ (withdrawal speed $=1 \mathrm{~mm} \mathrm{~s}^{-1}$ ). 
Processed from CF solution with residual time of $10 \mathrm{~s}$

(a)
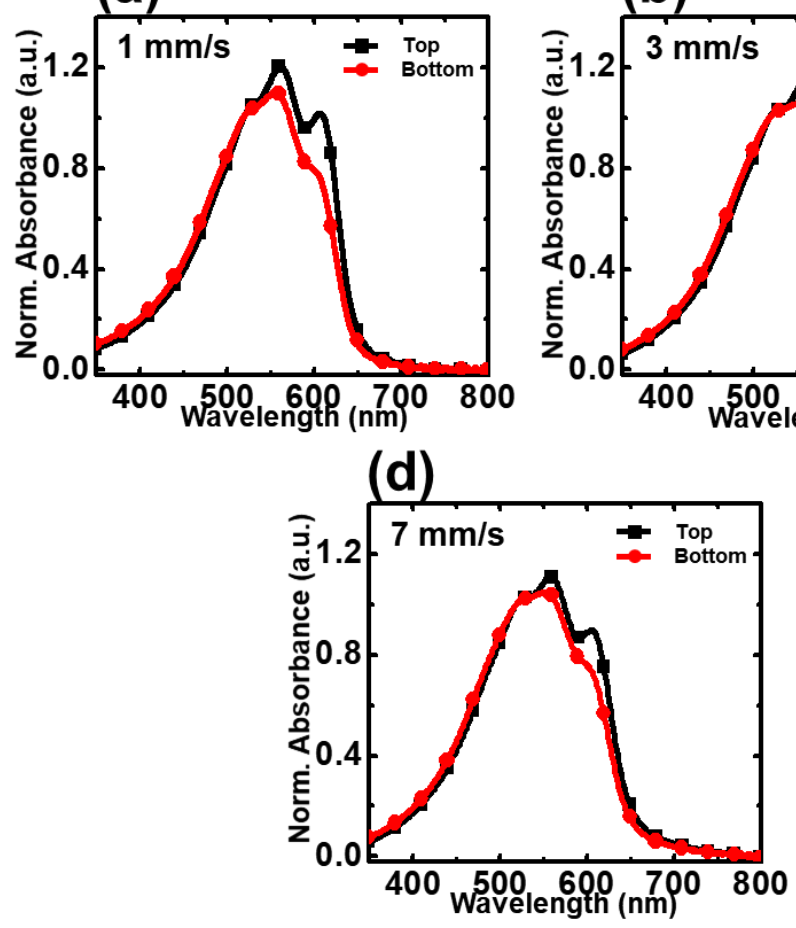

(b)

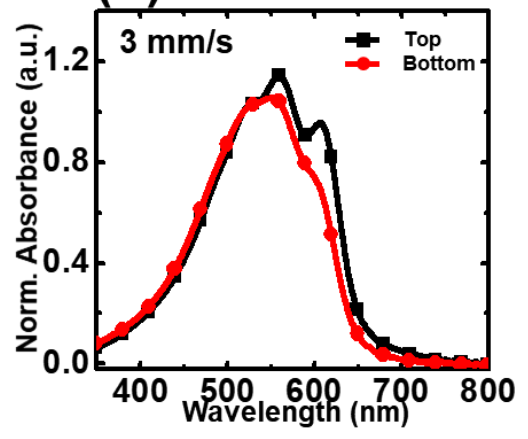

(c)

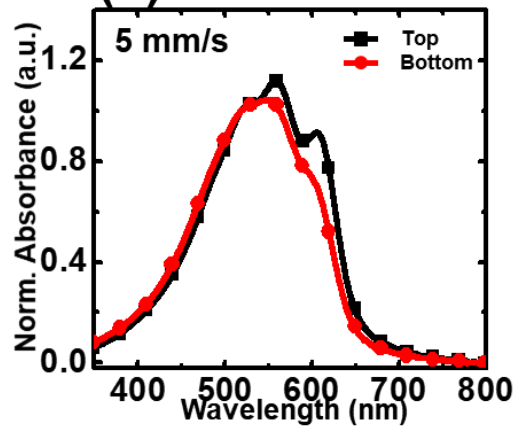

(e)

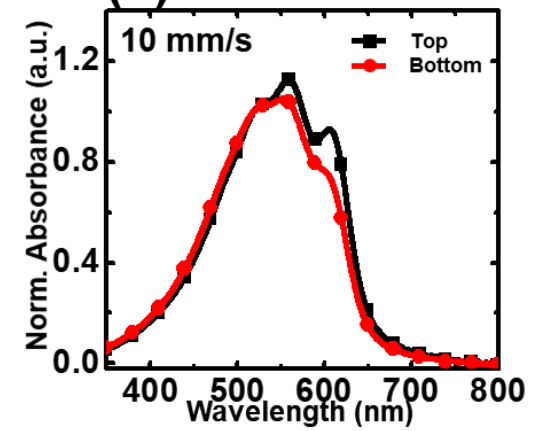

Figure S5. Normalized UV-vis absorption spectra of the P3HT thin films prepared by dip-coating in CF solution with a residual time of $10 \mathrm{~s}$ and withdrawn at (a) 1, (b) 3, (c) 5, (d) 7, and (e) 10 $\mathrm{mm} \mathrm{s}^{-1}$; at the designated position from the top to the bottom. 
Processed from CF solution with residual time of $20 \mathrm{~s}$

(a)
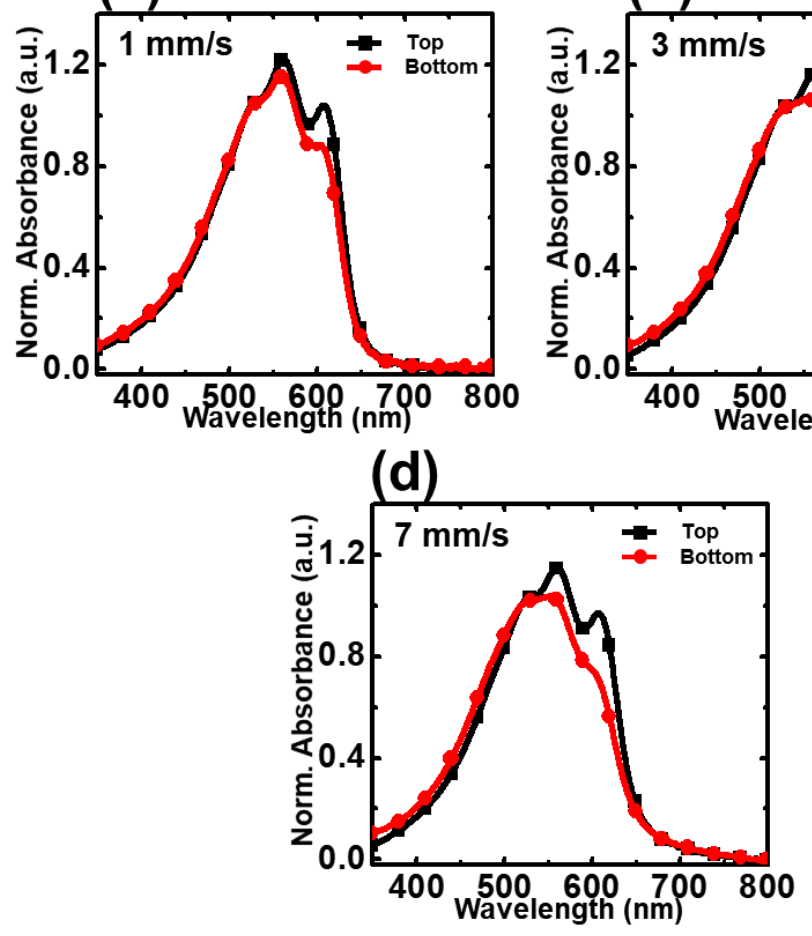

(b)

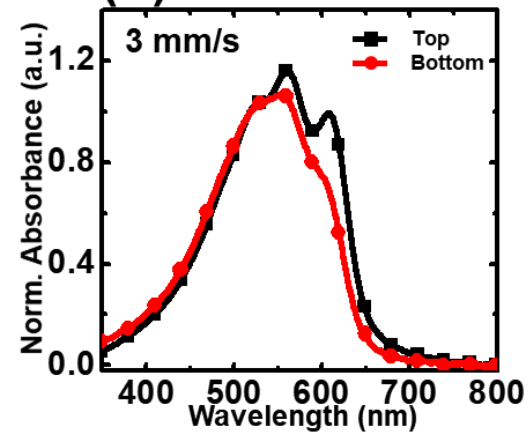

(c)

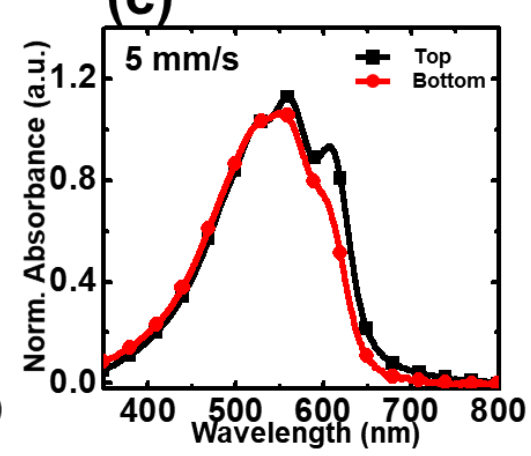

(e)

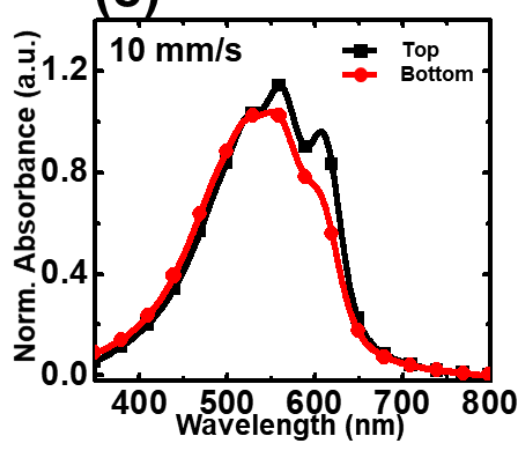

Figure S6. Normalized UV-vis absorption spectra of the P3HT thin films prepared by dip-coating in CF solution with a residual time of $20 \mathrm{~s}$ and withdrawn at (a) 1, (b) 3, (c) 5, (d) 7, and (e) 10 $\mathrm{mm} \mathrm{s}^{-1}$; at the designated position from the top to the bottom. 
Processed from CF solution with residual time of $30 \mathrm{~s}$

(a)
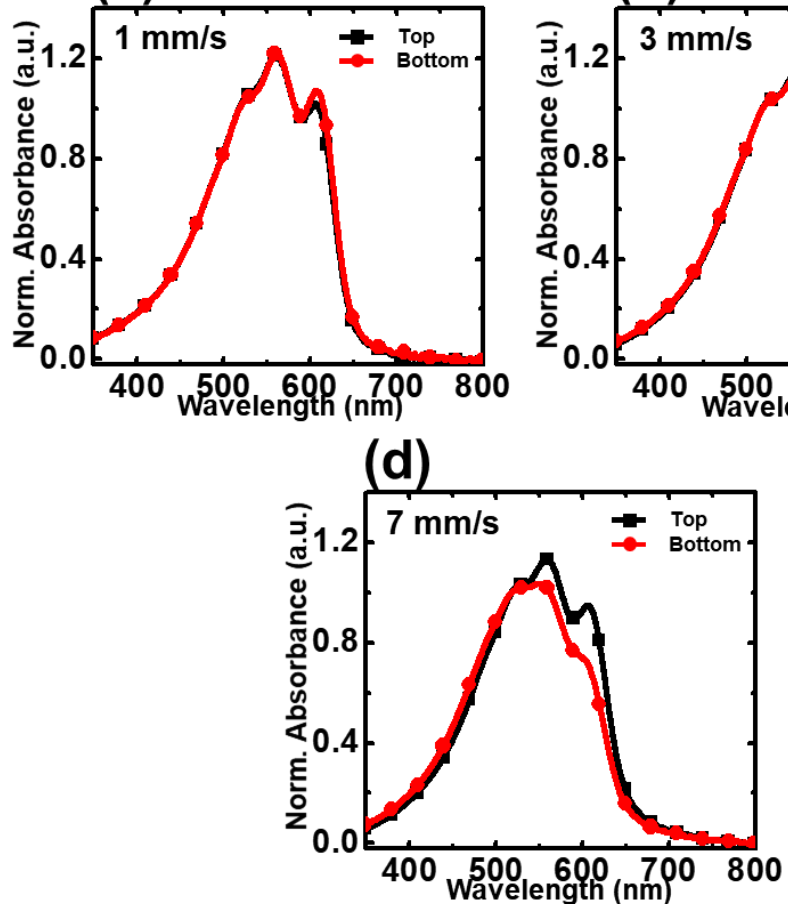

(b)

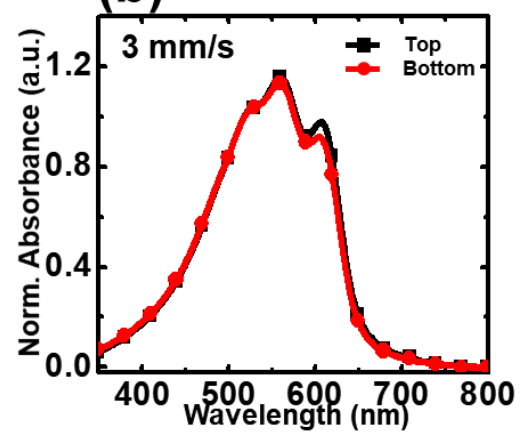

(c)

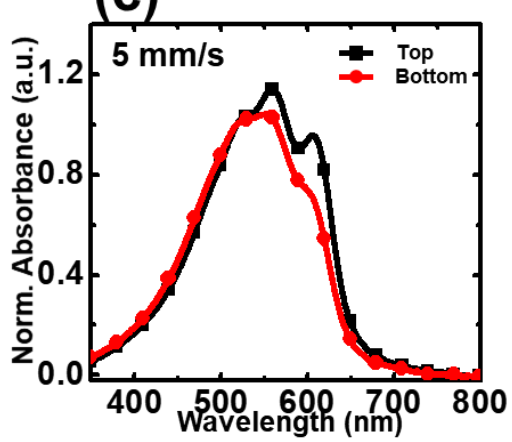

(e)

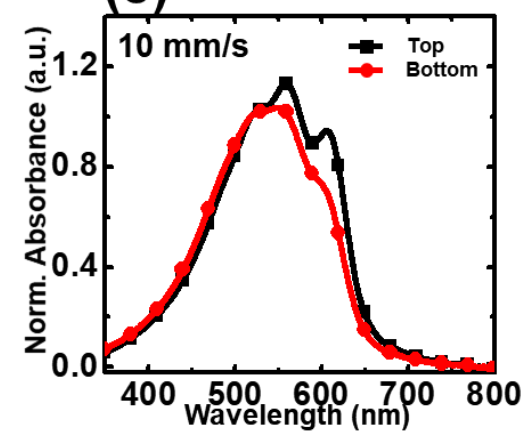

Figure S7. Normalized UV-vis absorption spectra of the P3HT thin films prepared by dip-coating in CF solution with a residual time of $30 \mathrm{~s}$ and withdrawn at (a) 1, (b) 3, (c) 5, (d) 7, and (e) 10 $\mathrm{mm} \mathrm{s}^{-1}$; at the designated position from the top to the bottom. 
Processed from CF solution with residual time of $40 \mathrm{~s}$

(a)

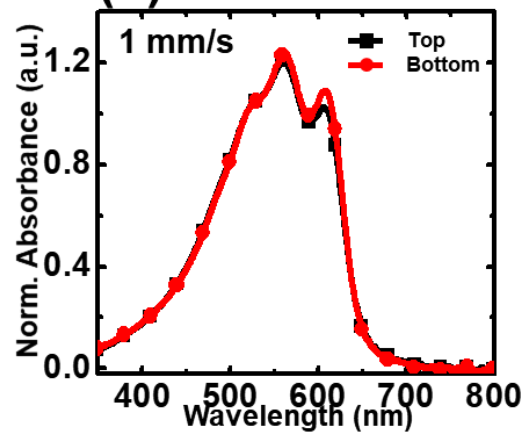

(d)

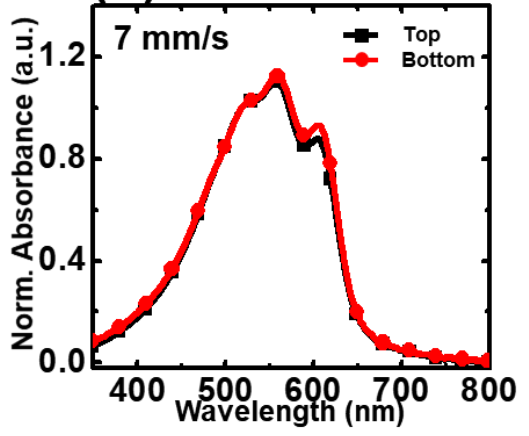

(b)

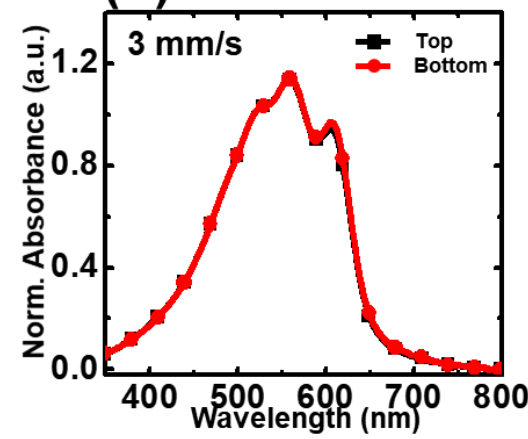

(c)

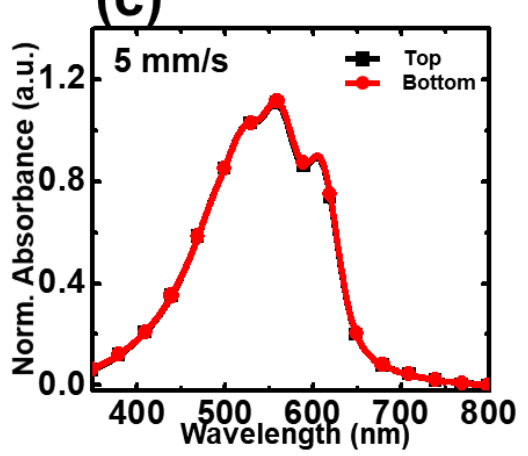

(e)

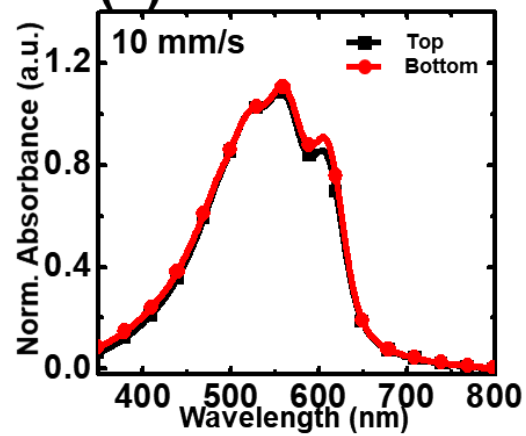

Figure S8. Normalized UV-vis absorption spectra of the P3HT thin films prepared by dip-coating in $\mathrm{CF}$ solution with a residual time of $40 \mathrm{~s}$ and withdrawn at (a) 1, (b) 3, (c) 5, (d) 7, and (e) 10 $\mathrm{mm} \mathrm{s}^{-1}$; at the designated position from the top to the bottom. 

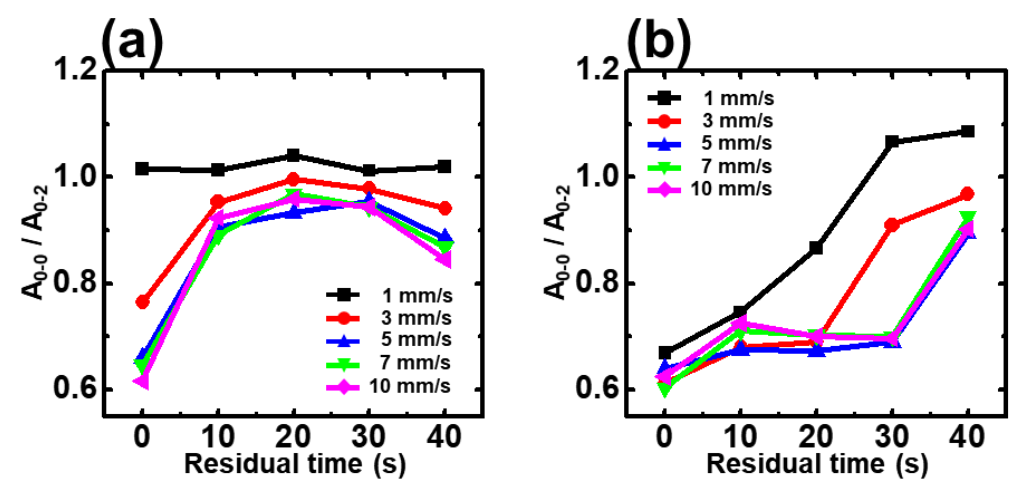

Figure S9. The ratios of the intensities of the (0-0) to (0-2) peaks of the P3HT thin films prepared by dip-coating in CF solution as a function of the withdrawal speed and the residual time in the dip-coating chamber. 

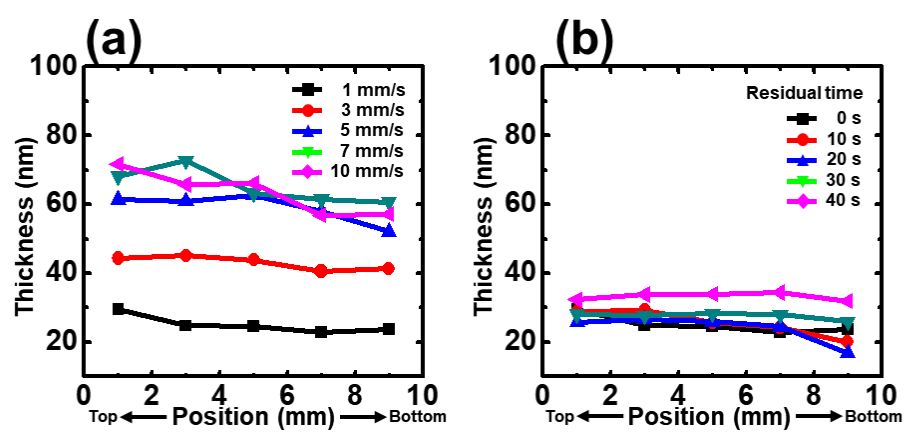

Figure S10. The thicknesses of the P3HT thin films prepared by dip-coating in CF solution as a function of the withdrawal speed and the residual time in the dip-coating chamber; at the designated position from the top to the bottom. 